\title{
Charging and heat collection by a positively charged dust grain in a plasma
}

\author{
Gian Luca Delzannd* and Xian-Zhu Tand \\ Theoretical Division, Los Alamos National Laboratory, Los Alamos, New Mexico 87545
}

(Dated: August 8, 2018)

\begin{abstract}
Dust particulates immersed in a quasineutral plasma can emit electrons in several important applications. Once electron emission becomes strong enough, the dust enters the positively charged regime where the conventional Orbital-Motion-Limited (OML) theory can break down due to potential well effects on trapped electrons. A minimal modification of the trapped-passing boundary approximation in the so-called $\mathrm{OML}^{+}$approach is shown to accurately predict the dust charge and heat collection flux for a wide range of dust size and temperature.

PACS numbers: 52.25.Dg, 52.27.Lw, 52.65.-y
\end{abstract}

The problem of the charging of a solid body in a plasma has a long history and various applications ranging from probes and spacecraft to planet formation to dusty plasmas in laboratory and space [1]. The body collects plasma particles and is often negatively charged owing to the higher electron mobility. In many instances, however, the body can emit electrons (via thermionic emission, photoemission and secondary electron emission) and become positively charged. Examples of such include spacecraft applications [2]; the moon [3]; meteoroids entering the Earth's atmosphere [4]; ionospheric rockets experiments [5]; dust particles in the solar wind [6], planetary rings [7], cometary environments 8], or magnetic fusion devices [9, 10]. Experiments showing the formation of ordered structures with positively charged dust are reported in laboratory [11, 12] and in microgravity [13]. Since charging is governed by the characteristic length of the body relative to the plasma Debye length or the electron gyroradius, there is no conceptual difference between the examples above and in what follows we will use the term dust broadly.

A charging theory is a necessary ingredient of any model of dust transport and destruction/survival in a plasma [4, 6, 7, 9, 14 19]. It calculates the dust charge/potential, momentum and heat collection due to the dust-plasma interaction. The most widely used charging theory is the Orbital-Motion-Limited (OML) theory [20], which leads to a simple nonlinear equation for the dust potential.

In this Letter, we use PIC simulations and theoretical analysis to show that OML can become inapplicable in the positively charged regime. It can completely miss the transition between negatively and positively charged dust (thus predicting a positive dust potential when simulations show a negative dust potential) and overestimates the power collected by the grain (up to a factor of 2 for the cases considered). This is due to the development of a non-monotonic potential (a potential well) near the grain. The fact that a potential well can exist near an electron emitting body is known [3, 21, 22]. However, this is the first study that illustrates the breakdown of OML in this regime. Moreover, this Letter presents a revised charging theory which is as simple as OML, recovers OML in the appropriate limits, but remains accurate when potential well effects are important.

We study the charging of a spherical dust grain of radius $r_{d}$ at rest in a collisionless, unmagnetized hydrogen plasma $\left(m_{e(i)}\right.$ and $T_{e(i)}$ are the electron (ion) mass and temperature, $n_{0}$ is the unperturbed plasma density away from the grain). The dust grain charges by collecting plasma and emitting electrons. The dynamics of the system is governed by the electric field created by the charged dust and a dynamical equilibrium is reached where the sum of all the currents on the dust surface is zero (floating condition).

In order to understand the OML limitations, we recall that the steady state of the system under consideration is completely determined by the orbital motion (OM) theory [23 25]. OM is based on the conservation of energy and angular momentum

$$
\begin{aligned}
v_{r}^{2}+v_{\theta}^{2}-\frac{2 e}{m_{e}} \phi_{d} & =v_{r}^{\prime 2}+v_{\theta}^{\prime 2}-\frac{2 e}{m_{e}} \phi(r), \\
m_{e} r_{d} v_{\theta} & =m_{e} r v_{\theta}^{\prime},
\end{aligned}
$$

and the conservation of the number of particles along characteristics in phase space (Liouville's theorem). Here we have introduced a spherical reference frame centered on the dust grain where $r$ is the radial distance and $v_{r}\left(v_{\theta}\right)$ is the radial (tangential) velocity of a particle, $e$ is the elementary charge, $\phi$ is the electrostatic potential, and $\phi_{d}=\phi\left(r_{d}\right)$. Equations (11) and (2) are for the emitted electrons, but similar relations hold for the background plasma. They can be combined into $v_{r}^{2}-U\left(r, v_{\theta}\right)=v_{r}^{\prime 2}$, stating the conservation of energy for a particle moving radially in the effective potential $U\left(r, v_{\theta}\right) \equiv \frac{2 e}{m_{e}}\left[\phi_{d}-\phi(r)\right]-\left[1-\left(\frac{r_{d}}{r}\right)^{2}\right] v_{\theta}^{2}=F_{E}-F_{C}$. The first (second) term of $U\left(r, v_{\theta}\right)$ is due to the electrostatic (centrifugal) force $F_{E}\left(F_{C}\right)$.

When electron emission is not dominant, the dust grain is negatively charged, $\phi(r)$ is monotonic, and all the emitted electrons leave the grain (labeled as passing electrons) and contribute to the emitted current. In this regime OML approximates OM by neglecting potential barriers 
to the ion motion associated with maxima of the ion effective potential [26, 27].

When electron emission is dominant, the dust grain is positively charged and $\phi(r)$ is non-monotonic: the slowest emitted electrons are attracted back to the grain creating a trapped electron population [22, 25]. Here 'trapped' refers to those emitted electrons that are recollected by the grain, and not to particles on a confined/bounded orbit as in probe theory [23, 27, 28]. The emitted electrons experience potential barriers to their motion: depending on $v_{\theta}$, the effective potential can have a maximum or be monotonically decreasing. The position of the maximum $r_{m}$ is given by $-\frac{e}{m_{e}} r_{m}^{3} \phi^{\prime}\left(r_{m}\right)=$ $r_{d}^{2} v_{\theta}^{2}$, which has one solution only for $r_{d} \leq r_{m} \leq r_{\text {min }}$ [the minimum of $\phi(r)$ is $\phi_{\min }=\phi\left(r_{\min }\right)$ ], namely when $\phi^{\prime}<0$. For $v_{\theta}=0$, the maximum is at $r_{m}=r_{m i n}$. One can therefore define a critical tangential velocity, $v_{\theta}^{*}=\sqrt{-\frac{e}{m_{e}} r_{d} \phi_{d}^{\prime}}$ [25], to characterize the electron orbits around the grain. For $v_{\theta}>v_{\theta}^{*}$ the effective potential is monotonically decreasing $\left(F_{C} \gg F_{E}\right)$ : all the emitted electrons leave the grain, irrespective of their radial velocity. For $v_{\theta}<v_{\theta}^{*}$, the effective potential has a maximum $\left(F_{E} \gtrsim F_{C}\right)$ : only those electrons with radial velocity $v_{r}>\sqrt{U\left(r_{m}\left(v_{\theta}\right), v_{\theta}\right)}$ leave the grain and contribute to the net current. Thus, the OM trapped/passing boundary (TPB) for evaluating the dust electron emission current is

$$
v_{r}^{2}=U\left(r_{m}\left(v_{\theta}\right), v_{\theta}\right)
$$

When the emitted electrons follow a Maxwellian distribution (representative of most applications) with temperature $T_{d}$ and thermal speed $v_{t h, t}=\sqrt{T_{d} / m_{e}}$, the implication of Eq. (3) can be elucidated for $v_{\theta}^{*} / v_{t h, t} \gg 1$. Most of the emitted electrons have velocity $v \lesssim v_{t h, t}$ and see a potential barrier located at $r_{m} \simeq r_{\min }$ corresponding to the TPB given by $v_{r}^{2}=U\left(r_{\min }, v_{\theta}\right)$, i.e.

$$
v_{r}^{2}+\left[1-\left(\frac{r_{d}}{r_{\min }}\right)^{2}\right] v_{\theta}^{2}=\frac{2 e}{m_{e}}\left(\phi_{d}-\phi_{\min }\right) .
$$

This produces an ellipsoid in velocity space with aspect ratio given by $1-\left(\frac{r_{d}}{r_{\text {min }}}\right)^{2}$.

Strictly speaking, OML always assumes a monotonic $\phi(r)$, so the trapping of emitted electrons is only possible if $\phi_{d}>0$. Adopting Sodha's formula for positively charged dust in vacuum [29], the OML TPB is

$$
v_{r}^{2}+v_{\theta}^{2}=\frac{2 e}{m_{e}} \phi_{d} .
$$

Contrasting Eqs. (4) and (5), it is clear that the OML TPB approximation is only resonable for $\left|\phi_{d}\right| \gg\left|\phi_{\min }\right|$ and $r_{d} \ll r_{\min }$. The discrepancy can lead to drastically different predictions for $\phi_{d}$ and the power $q_{e}$ collected by the dust grain from the background electrons, see Fig. 1,
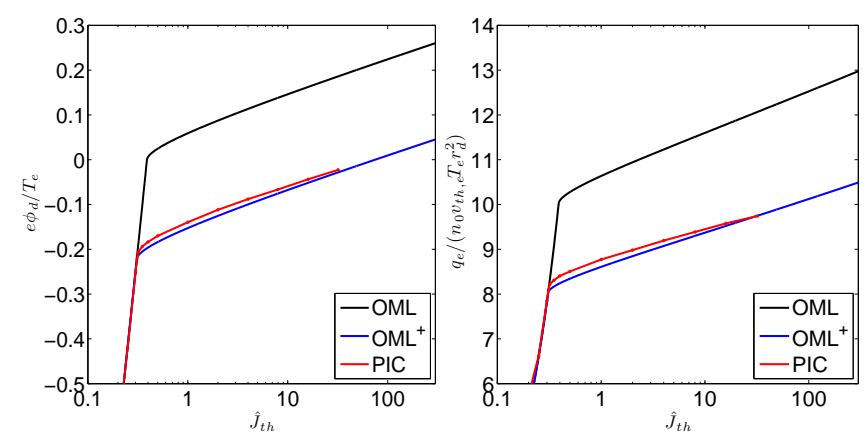

FIG. 1: Dust potential (left) and power collected by the dust from the background electrons (right) versus emitted current $\left[T_{d} / T_{e}=0.03, r_{d} / \lambda_{D e}=1\right]$.

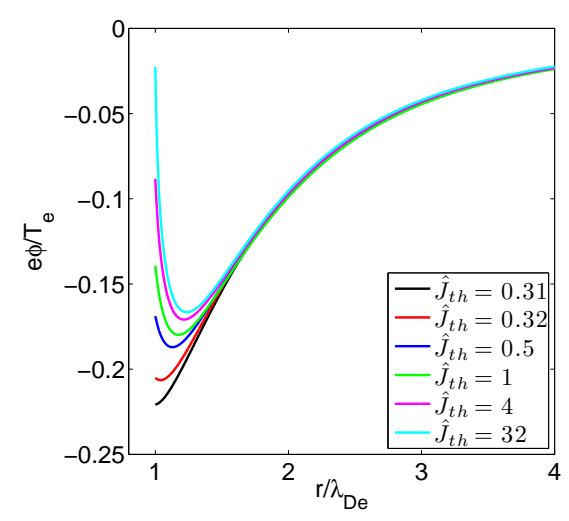

FIG. 2: Potential for various $\hat{J}_{t h}\left(T_{d} / T_{e}=0.03, r_{d} / \lambda_{D e}=1\right)$.

The OM result in Fig. 1 is obtained by a self-consistent electrostatic PIC simulation. This is for a perfectly conducting spherical dust grain as the inner boundary of the simulation domain, while the outer boundary is a concentric sphere of radius $R$. The dust grain emits electrons by thermionic emission, which are modeled with a Maxwellian distribution, $f_{t h}(\mathbf{v})=$ $2 \frac{m_{e}^{3}}{h^{3}} \exp \left(-\frac{m_{e} \mathbf{v}^{2}}{2 T_{d}}-\frac{W}{T_{d}}\right)$, with $h$ Planck's constant and $W$ the dust thermionic work function. For a negatively charged dust grain, the thermionic current density (normalized to $e n_{0} v_{t h, e}$ with $v_{t h, e}=\sqrt{T_{e} / m_{e}}$ ) is given by the Richardson-Dushman formula [30]

$$
\hat{J}_{t h}=\frac{J_{t h}}{e n_{0} v_{t h, e}}=\frac{4 \pi m_{e} T_{d}^{2}}{n_{0} v_{t h, e} h^{3}} \exp \left(-\frac{W}{T_{d}}\right) .
$$

The smallest time step of the simulations is $\Delta t \omega_{p e}=$ $0.0125\left(\omega_{p e}=\sqrt{e^{2} n_{0} /\left(\varepsilon_{0} m_{e}\right)}\right.$ with $\varepsilon_{0}$ vacuum permittivity), while other parameters are $R / \lambda_{D e}=10\left(\lambda_{D e}=\right.$ $\left.\sqrt{\varepsilon_{0} T_{e} /\left(n_{0} e^{2}\right)}\right), m_{i} / m_{e}=1836$, and $T_{i} / T_{e}=1$. More details on the simulation model can be found in Ref. [22].

The OML result in Fig. 1 is obtained using Eq. (6) when $\phi_{d}<0$, and by taking into account the trapping of emitted electrons when $\phi_{d}>0$ according to Eq. (5) [29]. The PIC and OML predictions are contrasted in 
Fig. 1 for $r_{d} / \lambda_{D e}=1$ and $T_{d} / T_{e}=0.03$. For reference, without thermionic emission the grain is negatively charged with good agreement between theory and simulations: $e \phi_{d}^{\mathrm{OML}} / T_{e} \simeq-2.50$ while $e \phi_{d}^{\mathrm{PIC}} / T_{e} \simeq-2.54$ 31]. The curves in Fig. [1 (left) exhibit the characteristic behavior associated with increasing thermionic emission: initially there is a sharp increase of the dust potential since the grain is becoming less negatively charged. All the emitted electrons leave the grain (hence the agreement with OML). At a critical current the curves bend since the dust grain is now positively charged and a population of trapped emitted electrons exists. PIC simulations show that the transition from negatively to positively charged grain occurs at negative dust potential $\left[e \phi_{d}^{* \mathrm{PIC}}\left(\hat{J}_{t h}^{*} \simeq 0.3\right) / T_{e} \simeq-0.22\right]$. Figure 1 (right) shows the (normalized) power collected by the dust from the background electrons $\hat{q}_{e}=q_{e} /\left(n_{0} v_{t h, e} T_{d} r_{d}^{2}\right)$. This is a particularly important quantity for dust survivability in a plasma, since positively charged grains are heated almost exclusively by the background electrons. As the negatively charged particle starts to emit thermionically, it reduces its charge and repels less background electrons. These electrons heat the dust particle, which emits more and further lowers its charge, and a positive feedback is established that can lead to dust destruction [9]. One can see in Fig. 1 that OML overestimates $\hat{q}_{e}$ by $\sim 30 \%$. Finally, the conventional relation between dust charge $Q_{d}$ and potential, $Q_{d}=4 \pi \varepsilon_{0} r_{d} \phi_{d}$, no longer holds (details will be presented elsewhere). As expected, these discrepancies are due to a deep and localized potential well (Fig. 2).

The equilibrium potential obtained by PIC can be used to check the emitted electrons TPB for OML and $\mathrm{OM}$ as shown in Fig. 3 for $\hat{J}_{t h}=2, r_{d} / \lambda_{D e}=1$ and $T_{d} / T_{e}=0.03$. For OM, $r_{m}\left(v_{\theta}\right)$ is calculated numerically and the TPB is given by Eq. (3). The approximate form, Eq. (4), is reasonably close in this range. For $\hat{J}_{t h}=2$ one has $\phi_{d}^{\mathrm{PIC}}<0$ so the OML approximation would have had passing electrons for the entire $\left(v_{r}, v_{\theta}\right)$ space. This cannot yield a solution since the OML currents cannot balance for the correct $\phi_{d}^{\mathrm{PIC}}$. Instead, OML forces an incorrect prediction of a positive $\phi_{d}^{O M L}$ in order to reduce (relative to $\hat{J}_{t h}$ ) the emitted electron current.

Can we anticipate when potential well effects are important and OML becomes inaccurate? This is set by the critical thermionic current $\hat{J}_{t h}^{*}$ corresponding to $Q_{d}=0$, below which OML is still accurate when $r_{d} \sim \lambda_{D e}$ [32]. We find the solution by solving Poisson's equation $\nabla^{2} \phi=\frac{e}{\varepsilon_{0}}\left[n_{e}^{O M L}(\phi)-n_{i}^{O M L}(\phi)+n_{t h}(\phi)\right]$. The OML electron density can be found in Refs. 26, 27] while a new expression for the ion density will be presented elsewhere

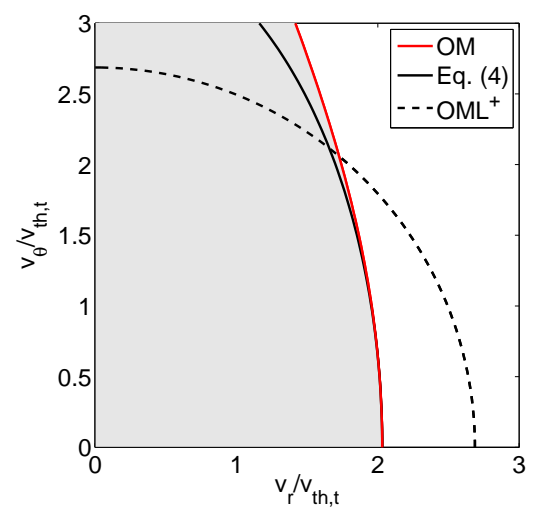

FIG. 3: Thermionic electrons trapped/passing boundary for $T_{d} / T_{e}=0.03, \hat{J}_{t h}=2$ and $r_{d} / \lambda_{D e}=1$.

[33. The density of the (passing) thermionic electrons is

$$
\begin{aligned}
& \frac{n_{t h}}{n_{0}}=\sqrt{\frac{\pi}{2}} \frac{\hat{J}_{t h}}{\sqrt{\frac{T_{d}}{T_{e}}}} \exp \left(e \frac{\phi-\phi_{d}}{T_{d}}\right)\left[1-\operatorname{erf} \sqrt{e \frac{\phi-\phi_{d}}{T_{d}}}-\right. \\
& \left.\exp \left[\frac{e\left(\phi-\phi_{d}\right)}{T_{d}\left(z^{2}-1\right)}\right] \frac{\sqrt{z^{2}-1}}{z}\left(1-\operatorname{erf} \sqrt{\frac{e\left(\phi-\phi_{d}\right) z^{2}}{T_{d}\left(z^{2}-1\right)}}\right)\right],
\end{aligned}
$$

where $z=r / r_{d}$. Poisson's equation is then solved with conditions $\phi\left(r_{d}\right)=\phi_{d}^{O M L}$ and $\phi^{\prime}\left(R \gg r_{d}\right)=0$, while the additional constraint $\left.Q_{d} \propto \phi^{\prime}\right|_{r_{d}}=0$ is used to determine $\hat{J}_{t h}^{*}$. For $T_{d} / T_{e}=0.03$ and $r_{d} / \lambda_{D e}=1$, we obtain $\hat{J}_{t h}^{*}=0.31$, in excellent agreement with Fig. 2. For their practical importance, we plot the contours of $\hat{J}_{t h}^{*}$ and $e \phi_{d}^{*} / T_{e}$ varying $T_{d} / T_{e}$ and $r_{d} / \lambda_{D e}$ in Fig. 4. The value of $\phi_{d}^{*}$ is representative of the importance of potential well effects: the higher $\left|e \phi_{d}^{*} / T_{e}\right|$, the more important these effects are. Figure 4 shows that for $r_{d} \ll \lambda_{D e}$ potential well effects are unimportant and OML is also a good approximation when $Q_{d}>0$. On the other hand, as $r_{d}$ increases these effects become important, more so if $T_{d} / T_{e}$ is small since the potential well is deeper and more localized. Conditions where potential well effects could be important are easily met in magnetic fusion applications [19] and can be met for mm-sized (and above) meteoroids entering the Earth's atmosphere [4].

Is it possible to develop an accurate approximation to $\mathrm{OM}$ as simple as OML when potential well effects are important? This is a challenge since Poisson's equation is not solved for $\phi(r)$ in an OML-like approach, so the promising approximation (4) cannot be deployed for lack of information on $r_{\min }$ and $\phi_{\min }$. Extending Sodha's idea 29], an OML-like TPB approximation would have $\phi_{d}$ in Eq. (5) replaced by $\phi_{d}-\phi_{d}^{*}$, since for $\phi_{d}>\phi_{d}^{*}$ the dust becomes positively charged and starts trapping some of the emitted electrons. So the revised OML-like TPB is

$$
v_{r}^{2}+v_{\theta}^{2}=\frac{2 e}{m_{e}}\left(\phi_{d}-\phi_{d}^{*}\right)
$$



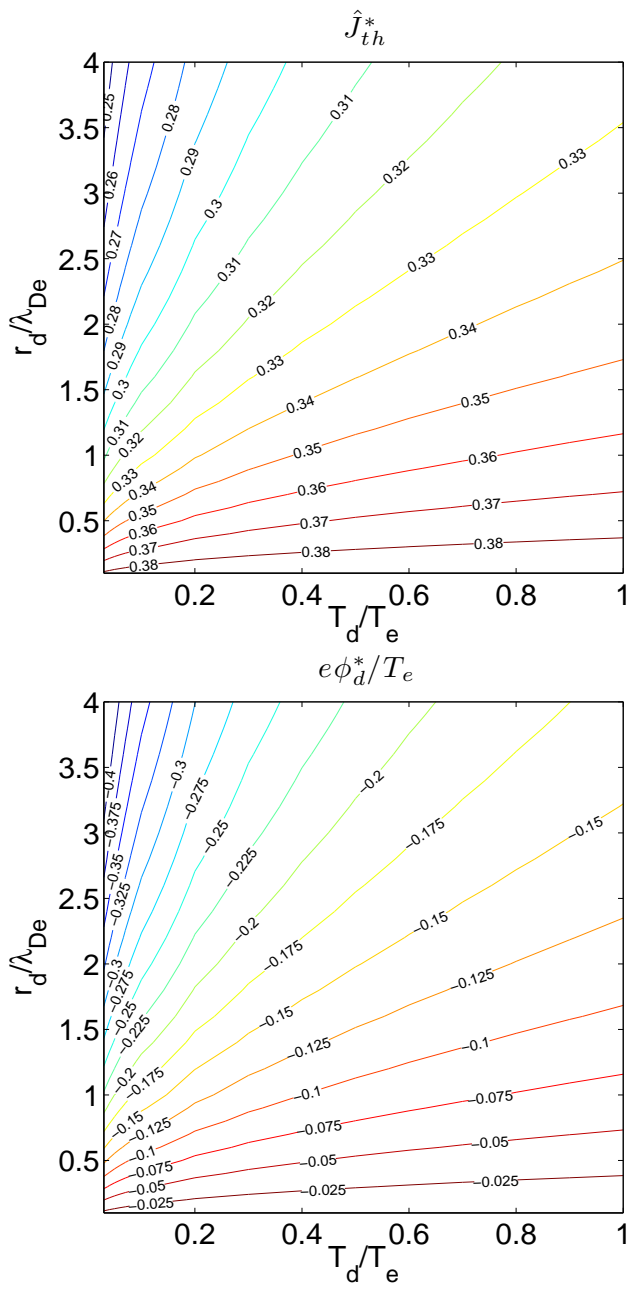

FIG. 4: Contours of $\hat{J}_{t h}^{*}$ and $e \phi_{d}^{*} / T_{e}$ varying $T_{d} / T_{e}$ and $r_{d} / \lambda_{D e}$.

From Fig. 3, one can see that Eq. (7) approximates the OM TPB in a least squares sense. From Eq. (7), the net thermionic current becomes:

$$
I_{t h}=4 \pi r_{d}^{2} J_{t h} \exp \left[-\frac{e\left(\phi_{d}-\phi_{d}^{*}\right)}{T_{d}}\right]\left[1+\frac{e\left(\phi_{d}-\phi_{d}^{*}\right)}{T_{d}}\right] .
$$

Thus, for $Q_{d}>0\left(\hat{J}_{t h}>\hat{J}_{t h}^{*}\right)$, Eq. (8) will be used to calculate the dust potential from the floating condition. The electron and ion background collection currents remain those of OML. We refer to approximation (8) as $\mathrm{OML}^{+}$, since it modifies OML only for $Q_{d}>0$.

The results from $\mathrm{OML}^{+}$are plotted in Fig. 1] where one can see that the agreement with PIC simulations is very good. For instance, for $\hat{J}_{t h}=32, \mathrm{OML}^{+}$gives $e \phi_{d} / T_{e} \simeq-0.028$, while $e \phi_{d}^{P I C} / T_{e} \simeq-0.023$. For comparison, $e \phi_{d}^{O M L} / T_{e} \simeq 0.19$. For higher $T_{d}$, for instance $T_{d} / T_{e}=0.5$, the potential well effect is not very important and both OML and $\mathrm{OML}^{+}$capture the dust potential reasonably (the relative error is less than $15 \%$, not shown). We have also investigated the effect of $r_{d}$ for
TABLE I: Parametric study increasing $r_{d}$ for $\hat{J}_{t h}=2$ and $T_{d} / T_{e}=0.03\left(R / \lambda_{D e}=20\right.$ for $\left.r_{d} / \lambda_{D e}>1\right)$.

\begin{tabular}{|c|c|c|c|c|c|c|}
\hline$r_{d} / \lambda_{D e}$ & $e \phi_{d}^{P I C} / T_{e}$ & $\hat{q}_{e}^{P I C}$ & $\hat{J}_{t h}^{*}$ & $e \phi_{d}^{*} / T_{e}$ & $e \phi_{d}^{\mathrm{OML}^{+}} / T_{e}$ & $\hat{q}_{e}^{\mathrm{OML}^{+}}$ \\
\hline 1 & -0.113 & 9.0 & 0.31 & -0.22 & -0.125 & 8.8 \\
2 & -0.208 & 8.1 & 0.27 & -0.33 & -0.228 & 8.0 \\
3 & -0.268 & 7.7 & 0.26 & -0.39 & -0.292 & 7.5 \\
4 & -0.309 & 7.4 & 0.24 & -0.44 & -0.336 & 7.2 \\
\hline
\end{tabular}

$\hat{J}_{t h}=2$ and $T_{d} / T_{e}=0.03$, as shown in Table I As expected, the dust potential is independent of $r_{d}$ in OML. In $\mathrm{OML}^{+}$, however, the $r_{d}$ dependence is taken into account through $\phi_{d}^{*}$, resulting in a remarkable agreement with PIC: in the cases studied the relative error between $\mathrm{OML}^{+}$and $\mathrm{PIC}$ is $\sim 10 \%$. Furthermore, the discrepancy between OML and PIC widens as $r_{d}$ increases, signaling that potential well effects are becoming more important [cf. Fig. 4. Table I] also shows $\hat{q}_{e}$ : for $r_{d} / \lambda_{D e}=4$, $\hat{q}_{e}^{O M L^{+}} \simeq \hat{q}_{e}^{P I C} \simeq 7.4$, while $\hat{q}_{e}^{O M L} \simeq 11$. Hence, in this case OML overestimates the dust collected power by roughly $50 \%$. In magnetic fusion energy applications such discrepancy can mean predicting dust destruction instead of survival, with strong implications for the safety and performance of the machine [19].

In conclusion, OML theory can break down in the positively charged regime for electron-emitting dust because it does not account for potential well effects on the TPB of the emitted electrons. Remarkably, once the critical dust potential $\phi_{d}^{*}$ at which the dust becomes positively charged is found by OML, a revised trappedpassing boundary for electron emission, Eq. (7), yields an $\mathrm{OML}^{+}$approximation that accurately predicts $\phi_{d}$ and dust power collection $q_{e}$ over a wide range of dust size $r_{d} / \lambda_{D e}$ and temperature $T_{d} / T_{e}$. With $\phi_{d}^{*}$ from Fig. 4 , $\mathrm{OML}^{+}$can be readily deployed by substituting Eq. (8) into the conventional OML theory.

This work was funded by the Laboratory Directed Research and Development (LDRD) program, U.S. Department of Energy Office of Science, Office of Fusion Energy Sciences, under the auspices of the National Nuclear Security Administration of the U.S. Department of Energy by Los Alamos National Laboratory, operated by Los Alamos National Security LLC under contract DEAC52-06NA25396.

* Electronic address: delzanno@lanl.gov

† Electronic address: xtang@lanl.gov

[1] P. K. Shukla and A. A. Mamun, Introduction to Dusty Plasma Physics (Institute of Physics Publishing, Philadelphia, 2001).

[2] E. Whipple, Reports on progress in Physics 44, 1197 (1981). 
[3] A. Poppe, J. S. Halekas, and M. Horányi, Geophysical Research Letters 38, L02103 (2011).

[4] G. Sorasio, D. Mendis, and M. Rosenberg, Planetary and Space Science 49, 1257 (2001).

[5] E. A. MacDonald, K. A. Lynch, M. Widholm, R. Arnoldy, P. M. Kintner, E. M. Klatt, M. Samara, J. LaBelle, and G. Lapenta, Journal of Geophysical Research: Space Physics 111, A12310 (2006).

[6] H. Kimura and I. Mann, Astrophys. J. 499, 454 (1998).

[7] M. Horányi and T. E. Cravens, Nature (London) 381, 293 (1996).

[8] B. Klumov, S. Vladimirov, and G. Morfill, JETP Letters 85, 478 (2007).

[9] R. D. Smirnov, A. Y. Pigarov, M. Rosenberg, S. I. Krasheninnikov, and D. A. Mendis, Plasma Physics and Controlled Fusion 49, 347 (2007).

[10] J. Vaverka, I. Richterová, M. Vyšinka, J. Pavlů, J. Šafránková, and Z. Němeček, Plasma Physics and Controlled Fusion 56, 025001 (2014).

[11] V. E. Fortov, A. P. Nefedov, O. F. Petrov, A. A. Samarian, and A. V. Chernyschev, Physics Letters A 219, 89 (1996).

[12] A. A. Samarian, O. S. Vaulina, A. P. Nefedov, V. E. Fortov, B. W. James, and O. F. Petrov, Phys. Rev. E 64, 056407 (2001).

[13] V. Fortov, A. Nefedov, O. Vaulina, A. Lipaev, V. Molotkov, A. Samaryan, V. Nikitskii, A. Ivanov, S. Savin, A. Kalmykov, et al., Journal of Experimental and Theoretical Physics 87, 1087 (1998).

[14] D. A. Mendis and M. Rosenberg, Annual Review of Astronomy and Astrophysics 32, 419 (1994).

[15] C. M. Ticos, Z. Wang, G. L. Delzanno, and G. Lapenta, Physics of Plasmas 13, 103501 (2006).

[16] X. Z. Tang and G. L. Delzanno, Journal of Fusion Energy 29, 407 (2010).

[17] M. Bacharis, M. Coppins, and J. E. Allen, Physics of Plasmas 17, 042505 (2010).

[18] S. Ratynskaia, L. Vignitchouk, P. Tolias, I. Bykov, B. H.
Bergsaker, A. M. Litnovsky, N. den Harder, and E. Lazzaro, Nuclear fusion 53, 123002 (2013).

[19] G. L. Delzanno and X. Z. Tang, Physics of Plasmas 21, 022502 (2014).

[20] H. Mott-Smith and I. Langmuir, Physical Review 28, 727 (1926).

[21] B. Thiebault, A. Hilgers, E. Sasot, H. Laakso, P. Escoubet, V. Genot, and J. Forest, Journal of Geophysical Research: Space Physics 109, A12207 (2004).

[22] G. L. Delzanno, G. Lapenta, and M. Rosenberg, Physical Review Letters 92, 350021 (2004).

[23] J. Laframboise, Tech. Rep., Toronto Univ. (Ontario). Inst. for Aerospace Studies (1966).

[24] R. Kennedy and J. Allen, Journal of Plasma Physics 69, 485 (2003).

[25] G. L. Delzanno, A. Bruno, G. Sorasio, and G. Lapenta, Physics of Plasmas 12, 62102 (2005).

[26] Ya. L. Al'pert, A. V. Gurevich, and L. P. Pitaevskii, Space Physics with Artificial Satellites (Plenum Press, New York, 1965).

[27] J. Allen, B. Annaratone, and U. de Angelis, Journal of Plasma Physics 63, 299 (2000).

[28] J. Goree, Phys. Rev. Lett. 69, 277 (1992).

[29] M. Sodha and S. Guha, Advan. Plasma Phys. 4, 219 (1971).

[30] N. W. Ashcroft and N. D. Mermin, Solid State Physics (Philadelphia, Saunders College, 1976).

[31] G. L. Delzanno, E. Camporeale, J. D. Moulton, J. E. Borovsky, E. A. MacDonald, and M. Thomsen, IEEE Transactions on Plasma Science 41, 3577 (2013).

[32] C. T. N. Willis, M. Coppins, M. Bacharis, and J. E. Allen, Plasma Sources Science and Technology 19, 065022 (2010).

[33] X. Z. Tang and G. L. Delzanno, Orbital-motion-limited theory of dust charging and plasma response, under review (2014). 